\title{
Benefit from thoracic radiotherapy in patients with extensive-disease small-cell lung cancer with elevated lactate dehydrogenase
}

This article was published in the following Dove Press journal:

OncoTargets and Therapy

I March 2016

Number of times this article has been viewed

\author{
Tao Qin ${ }^{1, *}$ \\ Ningning Zhou ${ }^{1, *}$ \\ Yin-duo Zeng ${ }^{2}$ \\ Xiaoxiao Dinglin ${ }^{2}$ \\ Yuanyuan Zhao' \\ Huai Liu ${ }^{3,4}$ \\ Likun Chen'
}

'Department of Medical Oncology, Sun Yat-sen University Cancer

Center, The State Key Laboratory of Oncology in South China, Collaborative Innovation Center for Cancer Medicine, ${ }^{2}$ Breast

Tumor Center, Sun Yat-sen University Sun Yat-sen Memorial Hospital, Guangzhou, Guangdong, ${ }^{3}$ Department of Radiotherapy, Hunan Cancer Hospital, ${ }^{4}$ Department of Radiotherapy, The Affiliated Cancer Hospital of Xiangya School of Medicine, Central South University, Key Laboratory of Translational Radiation Oncology, Changsha, Hunan, People's Republic of China

*These authors contributed equally to this work
Correspondence: Likun Chen Department of Medical Oncology, Sun Yat-sen University Cancer Center, The State Key Laboratory of Oncology in South China, Collaborative Innovation Center for Cancer Medicine, No 65I Dongfeng Road East, Guangzhou, Guangdong 510060, People's Republic of China

Tel +862087342475

Email chenlk@sysucc.org.cn
Background: High lactate dehydrogenase (LDH) is associated with a large tumor burden in extensive-disease small-cell lung cancer (ED-SCLC). This study evaluated the benefit of additional thoracic radiotherapy (TRT) in patients with ED-SCLC with elevated LDH.

Methods: We analyzed 94 patients with ED-SCLC and evaluated LDH at Sun Yat-sen University Cancer Center during the period between January 2000 and March 2010. Patients were divided into two groups according to whether TRT was received. Survival was evaluated by the KaplanMeier method and Cox's regression analysis.

Results: The median age of the 94 patients with ED-SCLC was 58.5 years. The main metastatic sites included the liver, bone, brain, and adrenal glands. The response rate in the TRT group was $46.9 \%$. There were 32 patients (34.04\%) receiving TRT and 5.3\% receiving prophylactic cranial irradiation. The median survival time reached 10 months $(95 \%$ confidence interval: $8.22,11.78$ months), and the 1-, 2-, and 5-year survival rates were $43.6 \%, 11.7 \%$, and $2.1 \%$, respectively. There was a significant difference in the median progression-free survival (PFS) and overall survival (OS) between the TRT group and the no TRT group (PFS: 9.0 months vs 6.0 months, $P=0.018$; OS: 13.0 months vs 9.0 months, $P=0.006$ ).

Conclusion: The use of TRT improves the survival of patients with ED-SCLC. Future studies should use the LDH level for categorizing patients for treatment.

Keywords: small-cell lung cancer, extensive stage, thoracic radiotherapy, LDH

\section{Introduction}

Small-cell lung cancer (SCLC) is an aggressive pulmonary tumor with a strong probability for early distant metastasis. ${ }^{1}$ The incidence of SCLC makes up $~ 15 \%$ of primary lung cancers. ${ }^{2,3}$ SCLC is highly sensitive to initial chemotherapy and radiotherapy; however, most patients eventually die of recurrent disease. ${ }^{4,5}$ Although there is a high response rate of up to $70 \%-90 \%$ in these patients, the median survival time of patients with extensive-disease (ED)-SCLC is $12-20$ months. ${ }^{6}$ SCLC generally has a more rapid doubling time, a higher growth fraction, and a greater propensity for early nodal and distant metastasis. ${ }^{7}$ The overall survival (OS) rate is low, with only $2 \%-10 \%$ of patients with either limited-disease (LD)-SCLC or ED-SCLC alive at 5 years. ${ }^{6}$ Patients with ED-SCLC were incurable with the goal of symptom palliation and/or survival prolongation. The median survival time of patients with ED-SCLC was 7 months as indicated by the International Association for the Study of Lung Cancer database. ${ }^{8}$ Combined chemotherapy with etoposide and cisplatin (EP) was the first-line treatment for patients with ED-SCLC. The median survival time without treatment was reported as 2-4 months. ${ }^{9}$ Furthermore, another study reported a 
7.1\% 5-year survival rate for patients with ED-SCLC undergoing additional thoracic radiotherapy (TRT). ${ }^{10}$

However, in 2010, the European Society for Medical Oncology (ESMO) consensus recommended that patients with ED-SCLC receive TRT. The value of radiation therapy for the local primary site of the tumor remains uncertain. A single-center randomized study indicated a 5.4\% 5-year improvement in a subgroup of patients with metastatic disease who had either a complete response (CR) or a partial response (PR) within the thorax and a complete remission of distant disease after initial chemotherapy with the use of concomitant TRT and chemotherapy versus chemotherapy alone. ${ }^{11}$ A 15 -year follow-up study showed that the median survival time of patients with ED-SCLC was 6.1 months and only $2 \%$ of the patients survived to 5 years. ${ }^{12}$ Furthermore, a Phase II study indicated that the median disease-free survival time was 4.2 months, and the median OS time was 8.3 months for those receiving TRT. ${ }^{13}$ Patients with elevated lactate dehydrogenase (LDH) have a poor prognosis compared to those with normal LDH, showing a reduction of 6 months in the OS. ${ }^{14}$ Moreover, a Phase II study showed that the antiCTLA4 monoclonal antibody ipilimumab, in combination with paclitaxel and carboplatin, improved immune-related progression-free survival (PFS); PFS and OS data were not yet reported. ${ }^{15}$ Many factors affect the outcomes of patients with ED-SCLC. Elevated LDH, disease stage, poor performance status (PS), and multiple metastatic sites are unfavorable prognostic factors in patients with ED-SCLC. ${ }^{16,17}$ However, whether TRT impacts the prognosis with unfavorable factors, such as elevated LDH, remains controversial. Therefore, we hypothesized that TRT improved the survival of patients with ED-SCLC with elevated LDH compared to those without TRT.

\section{Patients and methods}

\section{Patients}

This is a retrospective study that was approved by the Institutional Review Board of Sun Yat-sen University Cancer Center and decided that patients' consent was not necessary for this study. A total of 94 patients with ED-SCLC who received treatment at Sun Yat-sen University Cancer Center during the period between January 2000 and March 2010 were assessed. Patients were chosen according to the flow diagram (Figure 1).

ED-SCLC extends beyond the ipsilateral hemithorax and includes patients with malignant pleural or pericardial effusions defined by the Veterans Administration Lung Cancer Study Group as ED, which was more frequently used in clinical practice. Patients with ED-SCLC who were

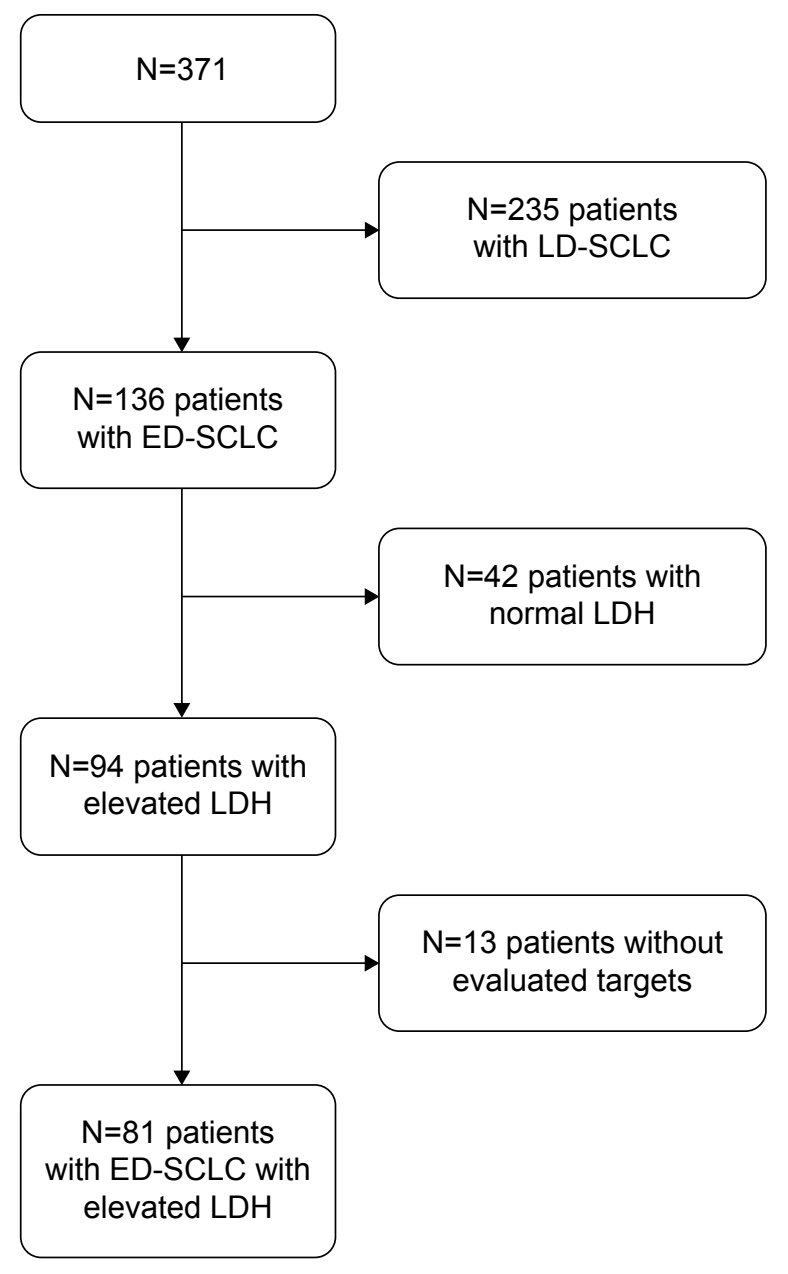

Figure I The flowchart of patient selection.

Abbreviations: ED-SCLC, extensive-disease small-cell lung cancer; LD-SCLC, limited-disease small-cell lung cancer; LDH, lactate dehydrogenase.

treated with TRT were enrolled. The inclusion criteria were as follows: known PS at the first diagnosis of ED, pathologically confirmed SCLC, known LDH, known metastatic sites, known survival status, and complete follow-up information. Patients who did not fulfill the inclusion criteria or those who had incomplete clinical data were excluded. We collected data on age, disease stage, date of initial diagnosis, date of death or last observation, survival time in months, PS score, serum level of $\mathrm{LDH}$ at diagnosis, metastatic sites, type and number of courses of first-line chemotherapy and subsequent lines of chemotherapy, key hematological and nonhematological toxicities, and result/benefit of first-line chemotherapy in terms of CR, PR, stable disease (SD), and progressive disease (PD).

\section{Treatment}

All patients received first-line chemotherapy, and 35 patients received concomitant or sequential TRT. Radiotherapy was predominately used as palliative care for patients with chest 
lesions, mediastinal, and supraclavicular lymph nodes. The total dose of TRT ranged from 40 Gy to 60 Gy at 1.8-2.0 Gy per fraction. Of the 94 patients, 13 received whole-brain radiation therapy. All patients received chemotherapy regimens containing cisplatin or carboplatin plus etoposide or sequential paclitaxel with cisplatin as the first-line chemotherapy.

\section{Follow-up}

Patients were evaluated every 2-3 months at outpatient clinics, where physical and imaging examinations were performed. All patients were followed up by telephone, with the calls by our hospital's follow-up office until November 31, 2013. The primary end point in this study was OS, defined as the length of time from the initiation of treatment after metastasis to death or the last follow-up.

Evaluation of efficacy was performed according to the criteria of the Response Evaluation Criteria in Solid Tumors (Version 1.0). Efficacy was classified as CR, PR, SD, and PD. PS evaluation was conducted using the Eastern Cooperative Oncology Group score.

\section{Statistical analysis}

Statistical analyses were performed using Statistical Package for the Social Sciences 16.0 software. For categorical variables, data were presented as the number (percent), and the chisquare test was used to test the relationship between two binary variables. Kaplan-Meier analysis with a log-rank test was performed to create cumulative survival curves. Univariate and multivariate Cox's proportional hazard analyses of OS were performed to identify prognostic clinicopathological factors for patients with ED-SCLC. Variables with a $P$-value $<0.05$ by univariate analysis were selected and evaluated by multivariate analysis. All statistical assessments were two sided, and statistical significance was set at $P<0.05$.

\section{Results}

\section{Patient characteristics}

The median age of all patients was 58.5 years. LDH was available for all 94 patients. The clinicopathological characteristics of the 94 patients with elevated LDH are shown in Table 1.

Table I Baseline patient characteristics at initial diagnosis

\begin{tabular}{|c|c|c|c|c|c|}
\hline Variables & $\mathbf{N}$ & $\%$ & TRT group & No TRT group & $P$-value \\
\hline \multicolumn{6}{|c|}{ Age of diagnosis } \\
\hline$<70$ years & 76 & 74.5 & 24 & 52 & 0.30 \\
\hline$\geq 70$ years & 18 & 25.5 & 8 & 10 & \\
\hline \multicolumn{6}{|l|}{ Sex } \\
\hline Male & 83 & 89.3 & 26 & 57 & 0.127 \\
\hline Female & 11 & 11.7 & 6 & 5 & \\
\hline \multicolumn{6}{|c|}{ ECOG PS score } \\
\hline $0-1$ & 87 & 92.6 & 30 & 57 & 1 \\
\hline 2 & 7 & 7.4 & 2 & 5 & \\
\hline \multicolumn{6}{|c|}{ Metastasis sites } \\
\hline Single & 45 & 47.9 & 14 & 31 & 0.565 \\
\hline Multiple & 49 & 52.1 & 18 & 31 & \\
\hline \multicolumn{6}{|c|}{ Liver metastasis $^{\mathrm{a}}$} \\
\hline Yes & 18 & 19.1 & 2 & 16 & 0.022 \\
\hline No & 76 & 80.9 & 30 & 46 & \\
\hline \multicolumn{6}{|c|}{ Brain metastasis $^{\mathrm{b}}$} \\
\hline Yes & 25 & 26.6 & 10 & 15 & 0.463 \\
\hline No & 69 & 73.4 & 22 & 47 & \\
\hline \multicolumn{6}{|c|}{ Bone metastasis } \\
\hline Yes & 13 & 13.8 & 3 & 10 & 0.369 \\
\hline No & 81 & 86.2 & 29 & 52 & \\
\hline \multicolumn{6}{|l|}{ LDH (U/L) } \\
\hline $226-450$ & 71 & 75.5 & 22 & 49 & 0.431 \\
\hline $45 \mathrm{I}-675$ & 12 & 12.8 & 6 & 6 & \\
\hline$>675$ & 11 & 11.7 & 4 & 7 & \\
\hline \multicolumn{6}{|l|}{$\mathrm{PCl}$} \\
\hline Yes & 5 & 5.3 & 3 & 2 & 0.208 \\
\hline No & 89 & 94.7 & 29 & 60 & \\
\hline
\end{tabular}

Notes: ancludes 17 patients with single liver metastasis. 'Includes ten patients with a single metastasis of the brain.

Abbreviations: ECOG, Eastern Cooperative Oncology Group; LDH, lactate dehydrogenase; PCl, prophylactic cranial irradiation; PS, performance status; TRT, thoracic radiotherapy. 


\section{Response}

Apart from 13 patients who had only bone metastasis without evaluated targeted sites, the response data were retrievable from 81 patients with recurrence who received systemic chemotherapy. The efficacy of first-line chemotherapy was as high as 56.4\%; CR was achieved for two patients $(2.5 \%)$, PR for $42(51.9 \%)$, SD for seven $(8.6 \%)$, and PD for 30 (37\%). In this study, additional TRT did not enhance the overall response rate in patients with ED-SCLC (46.4\% vs $58.5 \%, P=0.300$ ). The response frequency is shown in Table 2.

\section{Survival}

During the follow-up period, all the 94 patients died, with a median OS of 10 months (95\% confidence interval [CI]: $8.22,11.78$ months). The 1-, 2-, and 5-year survival rates for the 94 patients were $43.6 \%, 11.7 \%$, and $2.1 \%$, respectively (Figure 2). The 1-, 2-, and 5-year survival rates for the TRT group were $56.3 \%, 18.8 \%$, and $3.1 \%$, respectively, whereas the 1-, 2-, and 5-year survival rates for the no TRT group were $30.6 \%, 6.5 \%$, and $0 \%$, respectively.

There was a significantly increased PFS in the TRT group compared with that in the no TRT group (9.0 months vs 6.0 months, $P=0.018$, Figure $3 \mathrm{~A}$ ). Similarly, there was a significant difference in the median OS between the two groups (TRT group vs no TRT group, 13.0 months vs 9.0 months, $P=0.006$; 1 -year survival rate: TRT group vs no TRT group, $56.3 \%$ vs $30.6 \%$, Figure $3 \mathrm{~B}$ ). In addition, patients who presented with liver metastasis had a significantly shorter median OS than patients without liver metastasis ( 7 months vs 11 months, $P=0.019$, Figure $3 C$ ). However, patients with brain metastasis had a longer survival time compared to those without brain metastasis, with a median survival time of 10 months and 9 months, respectively, but this was not significant ( $P=0.102$, Figure $3 \mathrm{D}$ ). In these patients, a few had poor PS, and there was no significant difference in OS between the PS $\leq 1$ and PS $\geq 2$ subgroups ( $P=0.595$, Figure $3 \mathrm{E})$. The median survival time of female patients was longer than that of male patients

Table 2 Response rate of all patients after first-line chemotherapy

\begin{tabular}{llllll}
\hline $\begin{array}{l}\text { First-line } \\
\text { response }\end{array}$ & $\begin{array}{l}\text { TRT } \\
(\mathbf{n}=\mathbf{3 2})\end{array}$ & $\%$ & $\begin{array}{l}\text { No TRT } \\
(\mathbf{n}=\mathbf{6 2})\end{array}$ & $\%$ & P-value \\
\hline CR & 3 & 9.4 & $\mathrm{I}$ & 1.6 & 0.102 \\
PR & 12 & 37.5 & 37 & 59.7 & \\
SD & 3 & 9.4 & 5 & 8.1 & \\
PD & 14 & 43.8 & 19 & 30.6 & \\
\hline
\end{tabular}

Abbreviations: $C R$, complete response; $P D$, progressive disease; $P R$, partial response; $\mathrm{SD}$, stable disease, TRT, thoracic radiotherapy.

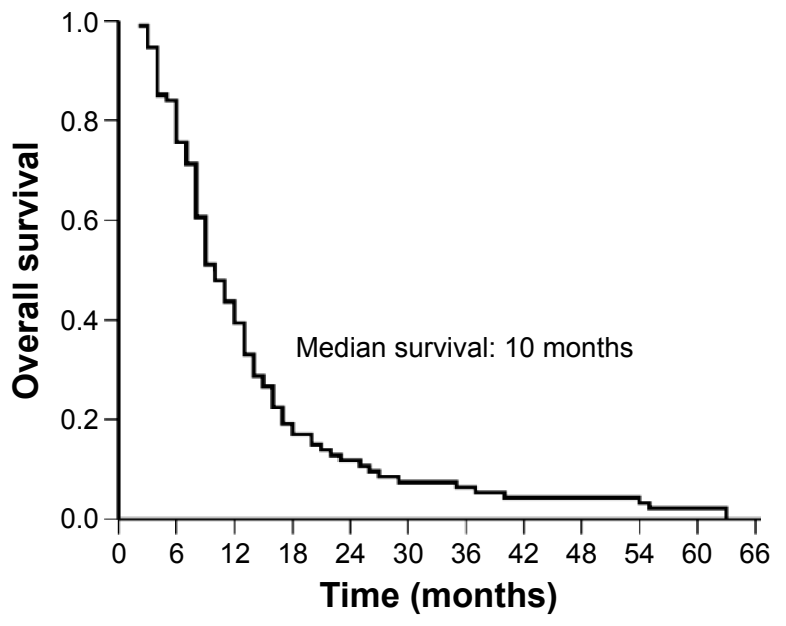

Figure 2 Overall survival time of 94 patients with elevated LDH. Abbreviation: LDH, lactate dehydrogenase.

in this study, but no significant difference was observed (median survival: 16 months vs 9 months, $P=0.197$, Figure $3 \mathrm{~F})$. Among males, there was a prolonged OS in the TRT group (12.0 months; no TRT group: 9.0 months, $P=0.038$, Figure 4A). There was no significant difference in OS between the two groups among females (TRT group: 17 months; no TRT group: 8.0 months, $P=0.250$, Figure 4B). Among patients without prophylactic cranial irradiation (PCI), there was a prolonged survival time in the TRT group (13.0 months; no TRT group: 8.0 months, $P=0.005$, Figure 4C). There was no significant difference in OS between the two groups among patients with PCI (TRT group: 17.0 months; no TRT group: 14.0 months, $P=0.946$, Figure 4D). In addition, we found no significant improvement in OS in the TRT group compared to that in the no TRT group in patients presenting with liver metastasis ( 7 months vs 7 months, $P=0.724$ ) or those presenting with brain metastasis (12 months vs 10 months, $P=0.862$ ).

Next, potential prognostic factors for 94 patients with ED-SCLC with an elevated LDH level were analyzed by univariate and multivariate Cox's proportional hazard regression analyses. Cox's proportional hazard regression analysis identified that TRT (yes vs no; $\mathrm{HR}=0.567 ; 95 \% \mathrm{CI}$ : 0.368-0.874), liver metastasis (yes vs no; $\mathrm{HR}=1.816 ; 95 \%$ CI: $1.069-3.083$ ), and metastasis site (single vs multiple; $\mathrm{HR}=0.57 ; 95 \% \mathrm{CI}: 0.368-0.881)$ were significant prognostic factors.

In the multivariate Cox's model, the factors favorably influencing survival were sensitivity to TRT and the number of metastatic sites (Table 3). However, age, PS, body mass index at initial diagnosis, PCI, brain metastasis, and liver metastasis were not significant prognostic factors. 

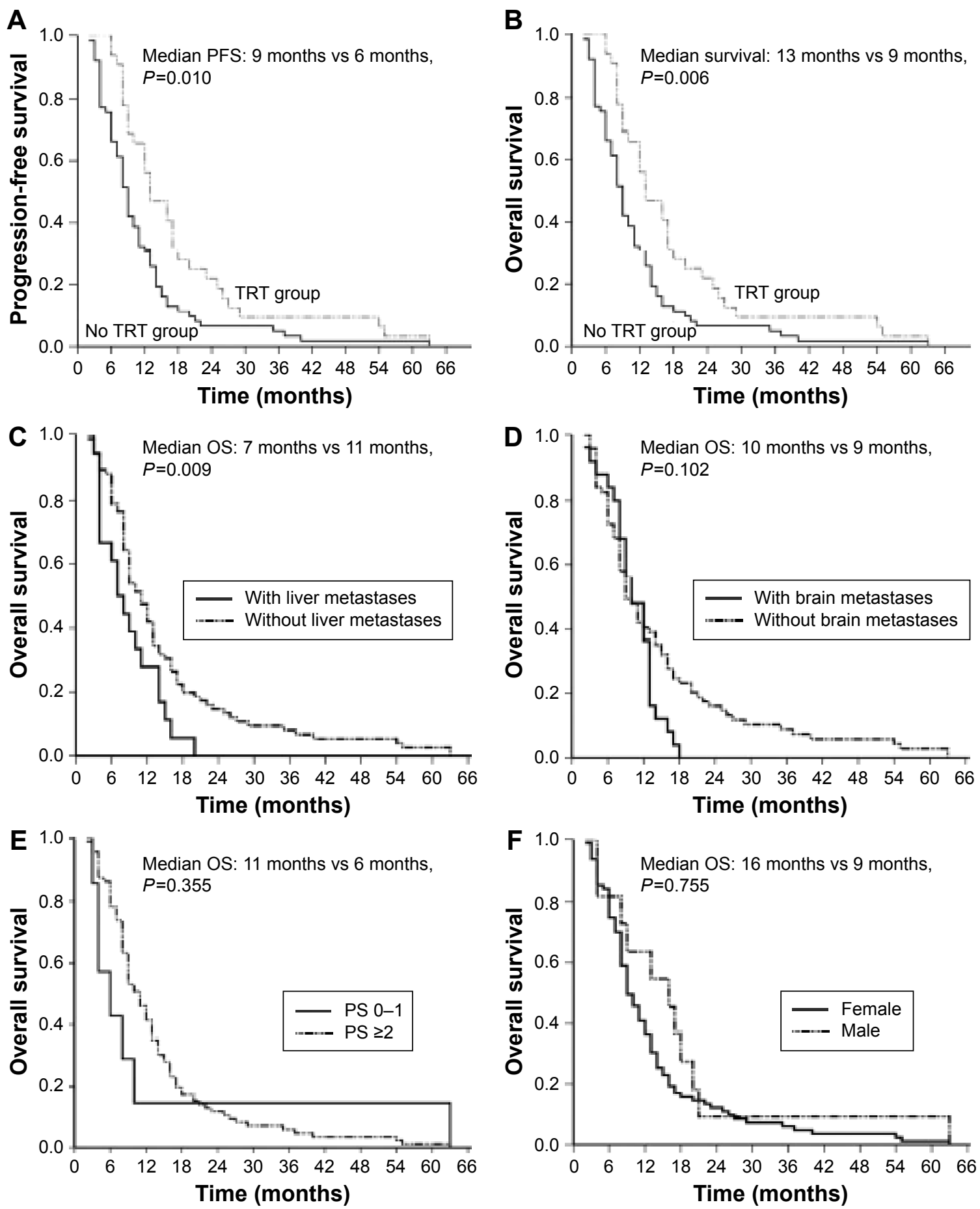

Figure 3 PFS of patients with or without TRT (A), OS (B), OS of patients with or without liver metastases (C), OS of patients with or without brain metastases (D), and OS of patients stratified by PS (E) and sex (F).

Abbreviations: OS, overall survival; PFS, progression-free survival; PS, performance status; TRT, thoracic radiotherapy.

\section{Toxicity}

No treatment-related deaths were found in these patients. No difference was found in terms of leukopenia between the TRT group and the no TRT group. In addition, other toxicities did not differ significantly between these groups. Acute reactions, including esophagitis and radiation pneumonitis, were found in patients who received TRT. All hematologic and nonhematologic toxicities are shown in Table 4.

\section{Discussion}

The present data demonstrated the significance of TRT as a strong independent factor in ED-SCLC in a contemporary 

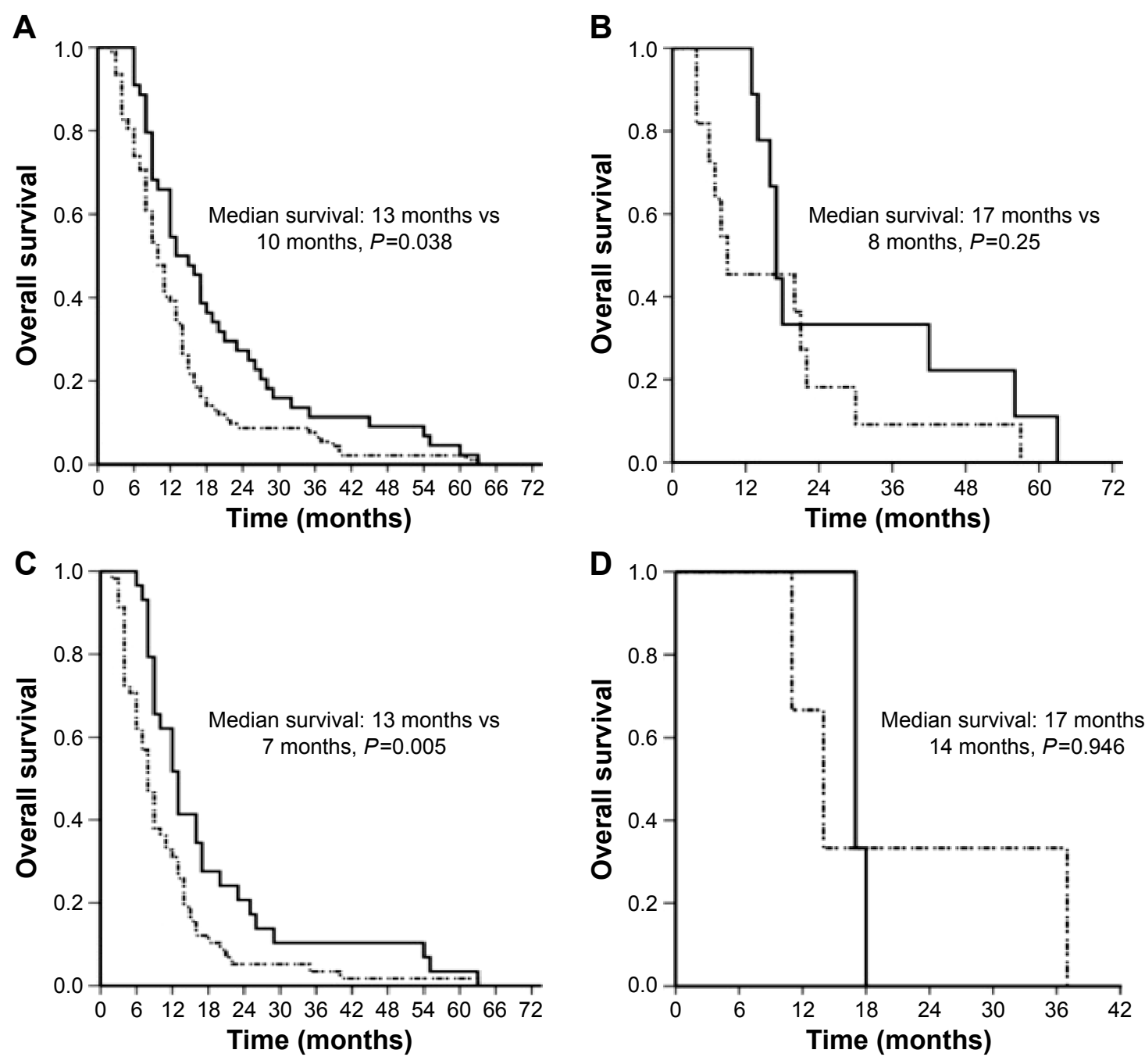

\section{$\because$ No TRT $\quad \sim$ TRT}

Figure 4 Comparison of OS between the TRT and no TRT groups according to sex and based on PCl: male (A), female (B), PCl (C), and no PCl (D). Abbreviations: OS, overall survival; $\mathrm{PCl}$, prophylactic cranial irradiation; TRT, thoracic radiotherapy.

Table 3 Multivariate analysis of prognostic factors by Cox's regression model

\begin{tabular}{|c|c|c|c|c|}
\hline \multirow[t]{2}{*}{ Variables } & \multirow[t]{2}{*}{ HR } & \multicolumn{2}{|c|}{ 95\% $\mathrm{Cl}$ for $\mathrm{HR}$} & \multirow[t]{2}{*}{$P$-value } \\
\hline & & Low & High & \\
\hline Sex (female vs male) & 0.906 & 0.455 & 1.801 & 0.777 \\
\hline TRT (yes vs no) & 0.569 & 0.344 & 0.941 & 0.028 \\
\hline $\mathrm{PCl}$ (yes vs no) & 0.755 & 0.314 & 1.816 & 0.531 \\
\hline WBRT (yes vs no) & 0.815 & 0.422 & 1.574 & 0.542 \\
\hline Metastasis site (multiple vs single) & 0.543 & 0.318 & 0.926 & 0.025 \\
\hline Liver metastasis (yes vs no) & 1.050 & 0.499 & 2.210 & 0.898 \\
\hline Brain metastasis (yes vs no) & 1.531 & 0.887 & $2.64 I$ & 0.126 \\
\hline $\mathrm{PS}(\leq \mathrm{I}$ vs $>\mathrm{I})$ & 1.028 & 0.457 & 2.313 & 0.946 \\
\hline Age $(>70$ years vs $\leq 70$ years $)$ & 1.293 & 0.847 & 1.973 & 0.234 \\
\hline $\mathrm{BMI}(\leq 18.5$ vs $>18.5)$ & 0.981 & 0.911 & 1.057 & 0.614 \\
\hline
\end{tabular}

Abbreviations: $\mathrm{BMI}$, body mass index; $\mathrm{Cl}$, confidence interval; HR, hazard ratio; $\mathrm{PCl}$, prophylactic cranial irradiation; PS, performance status; TRT, thoracic radiotherapy; WBRT, whole-brain radiotherapy treatment. patient population with elevated LDH at a single institution. In this study, the median survival time was 10 months and the 5 -year survival rate was $2.1 \%$ for those with elevated LDH. In addition, we note that using TRT improved the survival of patients with ED-SCLC with elevated LDH. Furthermore, we found that patients with elevated $\mathrm{LDH}$ receiving chest radiotherapy had longer PFS and OS compared to those not receiving TRT.

ED-SCLC accounts for a certain subgroup of SCLC, and chemotherapy is the standard treatment to control disease progression. This disease is generally chemo sensitive, and multi-agent chemotherapy has been effective in improving OS. A current review summarizes the evidence for the role of TRT as well as PCI in combination with chemotherapy in both 
Table 4 Adverse events summarized by treatment group

\begin{tabular}{llll}
\hline Toxicity (grades 3-4) & TRT group (\%) (n=32) & No TRT group (\%) (n=62) & P-value \\
\hline Leukopenia & $9(28.1)$ & I5 (24.2) & 0.679 \\
Thrombocytopenia & $\mathrm{I}(3.1)$ & $5(8.1)$ & 0.667 \\
Anemia & $\mathrm{I}(3.1)$ & $3(4.8)$ & $\mathrm{I}$ \\
Nonhematologic toxicity & & & $\mathrm{I}$ \\
$\quad \mathrm{I}$ Nausea & $\mathrm{I}(3.1)$ & $\mathrm{I}(1.6)$ & $\mathrm{I}$ \\
$\quad$ Vomiting & $\mathrm{I}(3.1)$ & $\mathrm{I}(1.6)$ & - \\
$\quad$ Fatigue & 0 & 0 & - \\
TRT-induced toxicity & & 0 & - \\
$\quad$ Esophagitis & $\mathrm{I}$ & 0 & \\
$\quad$ Pneumonitis & $\mathrm{I}$ & 0 & \\
\hline
\end{tabular}

Abbreviation: TRT, thoracic radiotherapy.

LD-SCLC and ED-SCLC. ${ }^{9,18}$ Chemotherapy is a mainstay of treatment for ED-SCLC, while the use of radiotherapy has been reserved for local symptom palliation only. Similar to the LD-SCLC, the EP regimen remains a standard of care and benefits patients with ED-SCLC. ${ }^{19,20}$ In addition, the role of TRT in combination with chemotherapy was also beneficial for patients with ED-SCLC. A small, single institution randomized trial demonstrated the value of consolidation TRT in patients receiving TRT, as they had a significantly increased 5 -year survival rate of 5.4\% compared to those treated with chemotherapy alone. ${ }^{11}$ Similarly, in this study, TRT had an important role in improving the survival of patients, even though all patients were found to have elevated LDH. National Comprehensive Cancer Network (NCCN) guidelines Version 2 published in 2014 recommended that consolidative TRT might be beneficial for selected patients with ED-SCLC who were responsive to chemotherapy based on the two studies. ${ }^{21,22}$ The RTOG 0937 study and the NTR1527 trial are ongoing to determine the value of TRT in ED-SCLC.

Prior studies have shown that the prognostic factors for SCLC were disease extent, PS, sex, and LDH. ${ }^{17,23}$ Female patients had a superior survival time compared to male patients in a previous study. ${ }^{17}$ According to sex, we found that TRT increased OS in male patients but not in female patients. This was partly because fewer female patients were included. In addition, no statistical significance was found in patients with PS 0-1 when compared to patients with PS 2. A study indicated that patients with elevated LDH demonstrated a short survival time in the ED subgroup when compared to patients with normal LDH. ${ }^{14}$ A previous study by $\mathrm{Zhu}$ et al found that median OS was 17 months, while the 2- and 5-year survival rates for patients receiving TRT were $35 \%$ and $7.1 \%$, respectively. ${ }^{10}$ Similarly, we also demonstrated that elevated LDH was associated with both short PFS and OS. However, our present survival data were shorter than the data reported by Zhu et al. The reason for this might be that all patients in this study presented with high LDH. However, this finding showed that patients with high LDH also benefited from TRT, with a higher 5-year survival rate reported by the study, which should be validated in a further study.

A meta-analysis showing a survival benefit with the use of PCI in patients with SCLC has also already disclosed this advantage for a subgroup of ED-SCLC. ${ }^{24,25}$ This study demonstrated a significant survival benefit with the use of PCI in ED-SCLC by reducing the risk of brain metastases, which led to an expectation of improved survival time with the use of TRT via a reduction in the rate of local relapses in chemotherapy responders at distant sites. Guidelines recommended that patients with either LD-SCLC or ED-SCLC who had a good response to initial therapy should receive PCI to decrease brain metastases and increase OS. ${ }^{19,26}$ PCI was applied with radiotherapy in the management of SCLC and showed an improved survival time with the use of PCI in both LD-SCLC and ED-SCLC. In this study, PCI did not improve PFS and OS; however, five patients with PCI had a favorable PFS with 11 months and a favorable OS with 17 months. In addition, similar to the use of TRT with systemic treatment, there were several debated issues with the use of PCI, such as timing, dose, and fractionation.

Different metastases, such as brain and liver metastases, also affected the survival time. ${ }^{27}$ Lekic et al reported that brain metastases were not a negative factor for OS if patients were treated appropriately. In this study, patients with liver metastases had an increased risk of death. However, patients with brain metastases had a similar prognosis compared to those without brain metastases. Here, whole-brain radiation therapy did not affect the survival time in both PFS and OS. 
In addition, the beneficial role of TRT was uncertain. We noted that patients without liver metastases benefited from TRT, but those with liver metastases did not. Similarly, no benefit from TRT was found in patients with brain metastases. We failed to conclude whether brain and liver metastases were independent factors for OS. Therefore, the presence of liver or brain metastases should be considered as potential risk factors when patients decided to receive TRT.

A previous study reported that the toxicity of grade 3 or 4 of leukopenia was more common in the TRT group compared to that in the no TRT group. ${ }^{10}$ However, grade 3 or 4 toxicity did not differ significantly between the TRT and no TRT groups in this study. The median age of all patients was 58.5 years in this study, which was less than the patients in the Zhu et al study, who had a median age of 61 years. The reasonable explanation might be that more patients who were younger than 65 years in the TRT group in this study.

\section{Conclusion}

In conclusion, the use of TRT improved the survival of patients with ED-SCLC. For unselected patients with ED-SCLC, TRT should be chosen based on personality. A future study should stratify patients by their LDH level.

\section{Acknowledgment}

This study was funded by a grant from the Science and Technology Planning Project of Guangdong Province (Grant No 2012B031800371).

\section{Disclosure}

The authors report no conflicts of interest in this work.

\section{References}

1. Rudin CM, Poirier JT. MYC, MAX, and small cell lung cancer. Cancer Discov. 2014;4(3):273-274.

2. Govindan R, Page N, Morgensztern D, et al. Changing epidemiology of small-cell lung cancer in the United States over the last 30 years: analysis of the surveillance, epidemiologic, and end results database. J Clin Oncol. 2006;24(28):4539-4544.

3. Jemal A, Siegel R, Xu J, Ward E. Cancer statistics, 2010. CA Cancer J Clin. 2010;60(5):277-300.

4. Murray N, Turrisi AT 3rd. A review of first-line treatment for small-cell lung cancer. J Thorac Oncol. 2006;1(3):270-278.

5. Hann CL, Rudin CM. Management of small-cell lung cancer: incremental changes but hope for the future. Oncology (Williston Park). 2008;22(13): 1486-1492.

6. Chua YJ, Steer C, Yip D. Recent advances in management of small-cell lung cancer. Cancer Treat Rev. 2004;30(6):521-543.

7. Tseng $\mathrm{HH}, \mathrm{He} \mathrm{B}$. Molecular markers as therapeutic targets in lung cancer. Chin J Cancer. 2013;32(2):59-62.

8. Hermes A, Waschki B, Gatzemeier U, Reck M. Characteristics, treatment patterns and outcomes of patients with small cell lung cancer a retrospective single institution analysis. Lung Cancer. 2011;71(3): 363-366.
9. van Meerbeeck JP, Fennell DA, De Ruysscher DK. Small-cell lung cancer. Lancet. 2011;378(9804):1741-1755.

10. Zhu H, Zhou Z, Wang Y, et al. Thoracic radiation therapy improves the overall survival of patients with extensive-stage small cell lung cancer with distant metastasis. Cancer. 2011;117(23):5423-5431.

11. Jeremic B, Shibamoto Y, Nikolic N, et al. Role of radiation therapy in the combined-modality treatment of patients with extensive disease small-cell lung cancer: a randomized study. J Clin Oncol. 1999;17(7): 2092-2099.

12. Gaspar LE, McNamara EJ, Gay EG, et al. Small-cell lung cancer: prognostic factors and changing treatment over 15 years. Clin Lung Cancer. 2012;13(2):115-122.

13. Yee D, Butts C, Reiman A, et al. Clinical trial of post-chemotherapy consolidation thoracic radiotherapy for extensive-stage small cell lung cancer. Radiother Oncol. 2012;102(2):234-238.

14. Hermes A, Gatzemeier U, Waschki B, Reck M. Lactate dehydrogenase as prognostic factor in limited and extensive disease stage small cell lung cancer - a retrospective single institution analysis. Respir Med. 2010; 104(12):1937-1942.

15. Reck M, Bondarenko I, Luft A, et al. Ipilimumab in combination with paclitaxel and carboplatin as first-line therapy in extensivedisease-small-cell lung cancer: results from a randomized, double-blind, multicenter phase 2 trial. Ann Oncol. 2013;24(1):75-83.

16. Planchard D, Le Pechoux C. Small cell lung cancer: new clinical recommendations and current status of biomarker assessment. Eur J Cancer. 2011;47(suppl 3):S272-S283.

17. Albain KS, Crowley JJ, LeBlanc M, Livingston RB. Determinants of improved outcome in small-cell lung cancer: an analysis of the 2,580patient Southwest Oncology Group data base. J Clin Oncol. 1990; 8(9):1563-1574.

18. Hu X, Bao Y, Chen YY, et al. [Efficacy of chemotherapy combined hyperfractionated accelerated radiotherapy on limited small cell lung cancer]. Ai Zheng. 2008;27(10):1088-1093.

19. Fruh M, De Ruysscher D, Popat S, et al; ESMO Guidelines Working Group. Small-cell lung cancer (SCLC): ESMO clinical practice guidelines for diagnosis, treatment and follow-up. Ann Oncol. 2013; 24(suppl 6):vi99-vi105.

20. Xiao X, Wang S, Xia S, et al. Retrospective study of irinotecan/cisplatin followed by etoposide/cisplatin or the reverse sequence in extensivestage small cell lung cancer. Onco Targets Ther. 2015;8:2209-2214.

21. Gandhi L, Johnson BE. Paraneoplastic syndromes associated with small cell lung cancer. J Natl Compr Canc Netw. 2006;4(6):631-638.

22. Kazarian M, Laird-Offringa IA. Small-cell lung cancer-associated autoantibodies: potential applications to cancer diagnosis, early detection, and therapy. Mol Cancer. 2011;10:33.

23. Foster NR, Mandrekar SJ, Schild SE, et al. Prognostic factors differ by tumor stage for small cell lung cancer: a pooled analysis of North Central Cancer Treatment Group trials. Cancer. 2009;115(12):2721-2731.

24. Stanic K, Kovac V. Prophylactic cranial irradiation in patients with small-cell lung cancer: the experience at the Institute of Oncology Ljubljana. Radiol Oncol. 2010;44(3):180-186.

25. Slotman B, Faivre-Finn C, Kramer G, et al; EORTC Radiation Oncology Group and Lung Cancer Group. Prophylactic cranial irradiation in extensive small-cell lung cancer. N Engl J Med. 2007;357(7):664-672.

26. Titulaer MJ, Wirtz PW, Willems LN, van Kralingen KW, Smitt PA, Verschuuren JJ. Screening for small-cell lung cancer: a follow-up study of patients with Lambert-Eaton myasthenic syndrome. J Clin Oncol. 2008; 26(26):4276-4281.

27. Lekic M, Kovac V, Triller N, Knez L, Sadikov A, Cufer T. Outcome of small cell lung cancer (SCLC) patients with brain metastases in a routine clinical setting. Radiol Oncol. 2012;46(1):54-59. 
OncoTargets and Therapy

\section{Publish your work in this journal}

OncoTargets and Therapy is an international, peer-reviewed, open access journal focusing on the pathological basis of all cancers, potential targets for therapy and treatment protocols employed to improve the management of cancer patients. The journal also focuses on the impact of management programs and new therapeutic agents and protocols on The manuscript management system is completely online and includes a very quick and fair peer-review system, which is all easy to use. Visit http://www.dovepress.com/testimonials.php to read real quotes from published authors.

Submit your manuscript here: http://www.dovepress.com/oncotargets-and-therapy-journal 